\title{
Correlation between the Authorship and Occupation of the Writer
}

\author{
Ujaala Jain ${ }^{1}$, Singh Monika ${ }^{2}$, Ahuja Pooja*3 and Dahiya MS ${ }^{4}$ \\ ${ }^{1}$ Student M.Sc Forensic Science programme, Institute of Forensic Science, Gujarat Forensic Sciences University, India \\ ${ }^{2,1}$ Student M.Sc Forensic Science programme, Institute of Forensic Science, Gujarat Forensic Sciences University, India \\ ${ }^{3}$ Assistant professor, Institute of Forensic Science, Gujarat Forensic Sciences University, India \\ ${ }^{4}$ Director, Institute of Forensic Science, Gujarat Forensic Sciences University, India
}

Submission: January 25, 2018; Published: February 05, 2018

*Corresponding author: Ahuja Pooja, Assistant professor, Institute of Forensic Science, Gujarat Forensic Sciences University, India, Email: pahuja159@gmail.com

Abstract

Handwriting is an art of penmanship which is acquired due to complex perceptual motor task. Its development skills begins with early scribbling and gradually develops into more intentional formation of shapes, patterns and letters that have meaning. In this paper, an attempt was made to ascertain the occupation of the writers by a statistical study of characteristic elements found in their handwriting. The targeted experimented group included bankers, doctors, teachers and unskilled workers irrespective of their age and gender. The successful intracomparison and inter-comparison according to the occupation of the writers were done to see the correlation of handwriting with the writer's occupation. A definite result obtained is that the handwriting of the writer and educational background are interrelated.

Keywords: Handwriting; Occupation; Legibility, Stylistics; Similarity; Chi-square

\section{Introduction}

Handwriting is considered as 'Brain-writing' because it is done due to the working of the brain sending signals to the writing instrument (hand of the writer). The precise ordering by brain and timing of the movements of arm and fingers determines the structure of the pattern. Handwriting is a foundation skill that needs to be developed early as it affects students' reading, language use, writing, and critical thinking [1]. Each one have a mental picture of what each letter looks like and then develop a motor plan to create that letter as it looks in our memory. The development of defined level handwriting skills (letter formation, sizing, spacing, alignment, and more) begins with being able to copy simple shapes. The brain cannot work as a computer so the letters cannot be replicated to what is seen, making every person's handwriting unique [2].

\section{Academic study of a forensic handwriting examination}

Class + Individual $=$ Academic. Features feature Identification [3]. Other than class and individual characteristics of handwriting, stylistics also plays a major role in handwriting examination. Stylistics is the study of individual characteristics in written language. The focus of forensic stylistics is written language and sometimes, spoken language represented in writing [4]. It covers the characteristics like arrangement (which is a group of habits that are influenced by writer's artistic ability, sense of proportion and instruction received), the placement of and balance of text-with respect to margins, the dimensions and uniformity of margins, class of allograph(letter formhand lettering or hand printing or block printing, composites of writing), connections (intraword i.e. between the letters of particular types, interword i.e. between words if when and where they occur), use of abbreviations-depends on writer's preference or the organization's policies and misspellings. Depending on the present condition of the writer, these internal and external factors may affect the handwriting for better or worse depending upon the presence or absence of the stimuli or factors. This can introduce temporary or permanent variation into a person's handwriting [5]. One such factor affecting the handwriting of an individual which is thoroughly discussed in this study is the 'occupation of the writer or the educational background' of the writer. With practice and skill, the execution of writing habits becomes more automatic, renders the writing process less subject to conscious control. So therefore, certain adaptations in handwriting do occur with time depending on the occupation of the writer [6]. For e.g. Bankers are more inclined towards typing on keyboard and therefore become less habitual of writing using pen and paper, thus a gradual decline in their 
writing do occur. So certain characteristics can be found similar in a group of writers of a particular occupation. On this basis, handwriting can be linked with the occupation of the writer.

Bhavana Desai, JL Kalyan [7] researched on the 'forensic examination of handwriting and signatures' and stated that the comparison of Handwriting and Signatures is dependent on class and individual characteristics of an individual. The pictorial appearance of the writing can change from childhood till adolescence but the individuality will not change. Therefore the focus and concentration of document examiner should be on the characteristics such as initial and final strokes, slant, alignment, t-crossing, i-dots, spacing etc. and tries to analyse handwriting or signatures. Khushbukumari and Prof. A K Kapoor studied the "handwriting characteristics variability among populations of Daman and Diu and concluded that variations are found in all population groups of Patel, muslim and macchi, and significant value found in word spacing, line spacing, letter spacing and capital word spacing, etc. of handwriting script in population groups. The study validated the individuality hypothesis with 95-percent confidence [8]. William L. Leaver wrote a book namely 'An introduction to forensic document examination' in which he discusses all the fundamentals of handwriting examination, covering the underlying principles and practical methods. This provides an overview of forensic document examination, examines the importance of interwriter $\&$ intrawriter variability, describes the techniques that are used to compare handwriting, demonstrates techniques that are used to compare printed documents, and examines the future of document examination in our new Digital World [9].

Van Drempt N, Mc Cluskey Lannin [10] reviewed 'the factors that influence adult handwriting performance' and stated that Age: Younger adults write more legibly and faster than older adults. Gender: Women write faster and more legibly than men. Pen grip: Grips other than the traditional dynamic tripod are functional, producing legible text in an acceptable time. Pen pressure: Pressure varies with different letters, words, text size, and speed and across a page of text. Error corrections and a mixed writing style occur in healthy adult handwriting. Denise Franz Sen and Aimee Stewart researched on the identifying the factors that contribute to hand writing problems experienced by students at a higher education institution in south Africa' determining the handwriting problems reported by 300 students at the University of the Witwatersrand when writing examinations. Students appear to have little understanding of their handwriting problems as those reporting that handwriting affected their ability to finish examinations were not those identified as having dysfunctional handwriting. Speed of writing was significantly affected by poor positioning of the paper, maintenance of grasp on the pen and accuracy when copying. Legibility in contrast was significantly worse due to holding the pen too close to the tip, pressure used when writing, deterioration or change in the writing errors related to missing words and spelling mistakes [11].
Baker examined the 'personal identification based on handwriting' discussing about elimination of the assumption that the written text is fixed by presenting a novel algorithm for automatic text-independent writer identification. Given that the handwriting of different people is often visually distinctive, they have taken a global approach based on texture analysis, where each writer's handwriting is regarded as a different texture [12]. Akoria $\mathrm{OA}$ researched on the 'prescription writing in public and private hospitals in benin city, Nigeria: the effects of an educational intervention' stating about the poor handwriting of doctors where the writing a prescription is a vital part of the process of rational therapeutics; a badly written prescription could undermine a clinical consultation. Poor quality of prescriptions was blamed partly on heavy workload and nonavailability of prescription order blanks. Prescriptions lacked details and most were not clearly legible. Intervention resulted in modest changes, which in public hospitals were more significant among doctors who had group seminars [13]. Steve Graham, Naomi Weintraub and Virginiaw examined 'the relation between handwriting style and speed and legibility' and concluded that the writing samples of the students who used a mixed style was faster than the handwriting of the students who used either printed or cursive handwriting exclusively. In addition, papers written with mixed mostly cursive letters generally received higher ratings for legibility than papers written with other three styles. There were no difference between manuscript and cursive in terms of speed or legibility [14].

\section{Methodology}

\section{Sample collection}

For this study, people working as unskilled workers, Bankers, Teachers, and Doctors were targeted for the sample collection based on different educational backgrounds. All the samples were collected from Guru Gram (Haryana) and Gandhinagar (Gujarat). Total 80 samples using blue ball pen were prepared, which includes 20 samples per occupation. All participants belonged to different geographical area but were living in Guru Gram and Gandhinagar for their earning while some of them were localities. All the handwriting samples were collected irrespective of the 'Age' and 'Gender' of the participants. The unskilled workers included carpenters, milkmen, security guards, shopkeepers, etc. The samples of teachers were taken from $1^{\text {st }}$ to $5^{\text {th }}$ grades teachers belonging to different schools. Similarly the samples of different bankers were taken from different branches of SBI bank with different working experiences. For the handwriting samples of doctors, prescription pages were used.

The figures below are the illustrations of samples that were collected from the volunteers of all the four targeted population: (Figures 1-4). The type of lettering is 'printed'. The legibility, speed, skills, spacing, alignment, rhythm and line quality is very low. All the characteristics observed like speed, skill, rhythm, alignment, line quality and legibly are medium in this writing. The type of lettering is 'cursive'. The legibility is high but the 
speed, skill and rhythm is medium. There is regular spacing Legibility is very low whereas speed, skill and rhythm are high.

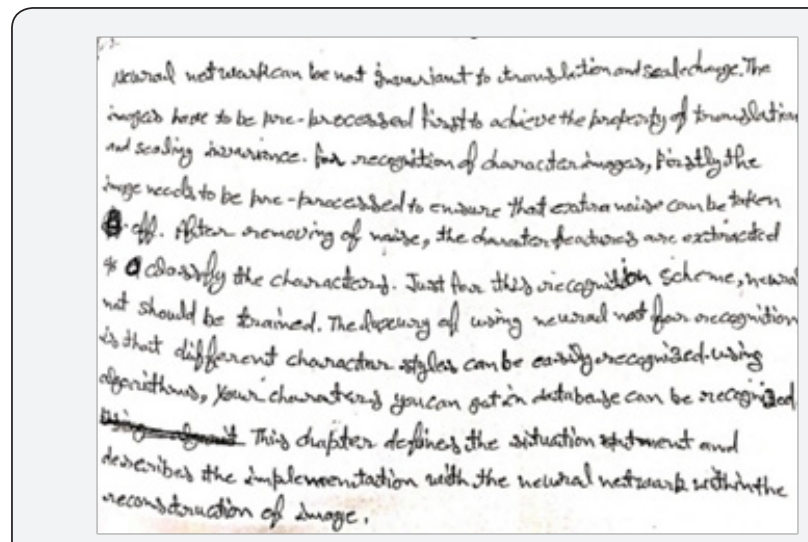

Figure 1: Unskilled worker's handwriting sample.

The type of lettering is 'printed'. The legibility, speed, skills, spacing, alignment, rhythm and line quality is very low.

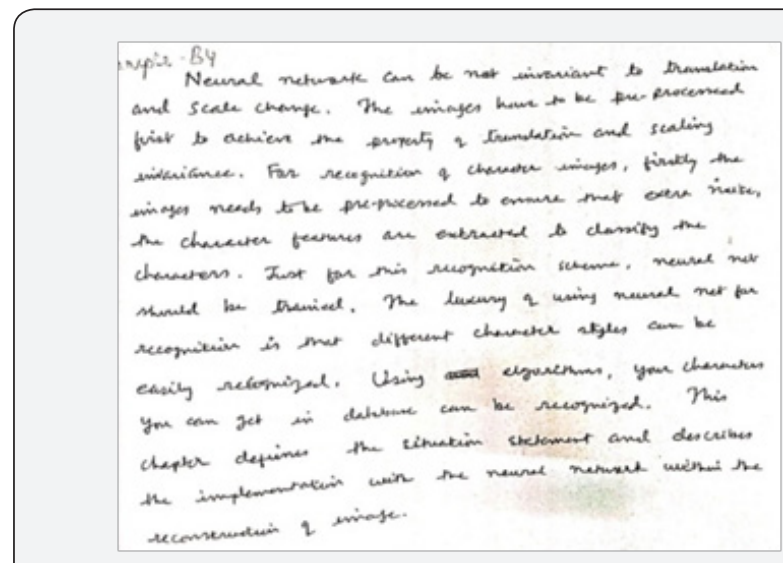

Figure 2: Banker's handwriting sample.

All the characteristics observed like speed, skill, rhythm, alignment, line quality and ligibily are medium in this writing.

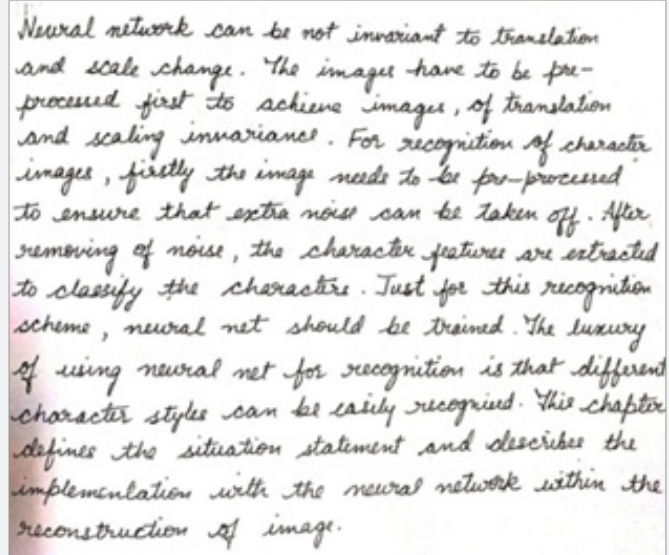

Figure 3: Teacher's handwriting sample.

The type of lettering is 'cursive'. The legibility is high but the speed, skill and rhythm is medium. There is regular spacing.

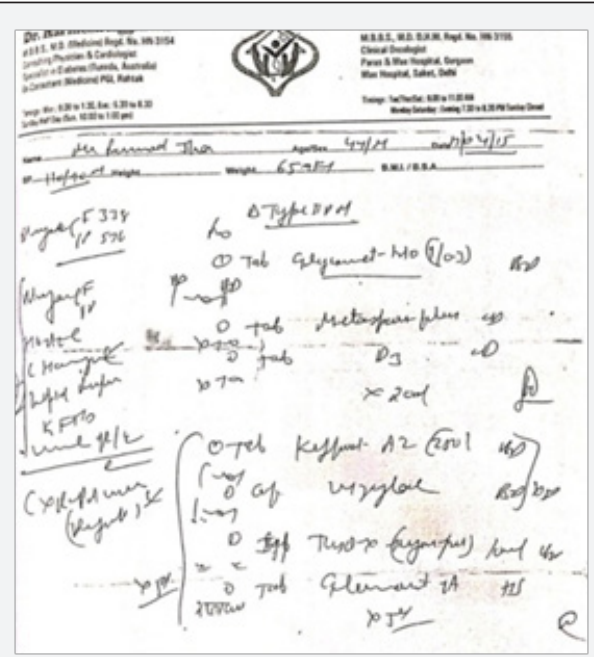

Figure 4: Teacher's handwriting sample.

Legibility is very low whereas speed, skill and rhythm is high.

\section{Methodology}

\section{Intra-Comparison}

The handwriting samples of one occupation were compared among themselves based on the general as well as individual handwriting characteristics to know the degree of similarity present in writers of a particular occupation.

\section{Inter-Comparison}

Each occupation was compared with other occupation to see the major differences in the general and individual characteristics of handwriting. For the calculation of the characteristics, the number of characteristics similar were counted for one occupation and filled in the table and this was repeated for each occupation.

\section{Degree of similarity}

After the intra comparison and inter comparison, calculating the percentage of similar characteristic in general to find the degree of similarity found in a particular occupation. To calculate percentage:

Similarly the degree of similarity was calculated for each characteristic for each occupation and the data was filled in the table.

\section{Chi-Square Test}

Chi square test was done using online chi- square test calculator to find the chi value of the both general and individual characteristics in order to find the eligibility of a particular characteristic to be able to differentiate between different occupations.

\section{Results and Discussions}

Tables 1 \& 2 inter-comparison of class and individual characteristics of different occupations (Depending on the 
sample size (80), each characteristic for each occupation is observed individually and then the inter-comparison of such characteristics is done to see the similarities and dissimilarities between the writing of people indulged in different occupations) The characteristics such as speed, skill and rhythm were found highest in doctors whereas legibility was found lowest. The legibility was found highest in teachers and speed, skill and rhythm were medium. The characteristics spacing (between letters, words and line) was found to be more regular as compared to doctors, bankers and unskilled workers. The characteristics such as legibility, speed, skill, spacing (between letters, words and line), alignment to baseline, rhythm and line quality was found low in unskilled workers. The characteristics legibility, speed, skill, spacing (between letters, words and line), alignment to baseline, rhythm and line quality was found medium in the handwriting samples of bankers. The type of lettering in case of unskilled workers was found to be majorly printed while majorly cursive in teacher's handwriting samples $80 \%$ and $85 \%$ respectively. The legibility of handwriting samples were found similar in case of unskilled workers and bankers i.e. medium $85 \%$ and $80 \%$ respectively. Size of handwriting samples was found similar in all the four occupations.

Table 1: inter-comparison of class and individual characteristics of different occupations.

\begin{tabular}{|c|c|c|c|c|}
\hline Characteritics & Unskilled workers & Teachers & Bankers & Doctors \\
\hline \multicolumn{5}{|c|}{ General Characteristics } \\
\hline \multirow{3}{*}{ Type of lettering } & Printed $(16 / 20)$ & Printed $(2 / 20)$ & Printed $(5 / 20)$ & Printed $(0 / 20)$ \\
\hline & Mixed (4/20) & Mixed (1/20) & Mixed (7/20) & Mixed $(9 / 20)$ \\
\hline & Cursive $(0 / 20)$ & Cursive $(17 / 20)$ & Cursive $(8 / 20)$ & Cursive $(11 / 20)$ \\
\hline \multirow{3}{*}{ Legibility } & Good $(0 / 20)$ & Good $(20 / 20)$ & Good $(0 / 20)$ & Good $(0 / 20)$ \\
\hline & Medium $(17 / 20)$ & Medium $(0 / 20)$ & Medium $(20 / 20)$ & Medium (2/20) \\
\hline & Poor $(3 / 20)$ & Poor $(0 / 20)$ & Poor $(0 / 20)$ & Poor $(18 / 20)$ \\
\hline \multirow{3}{*}{ Speed } & High $(0 / 20)$ & High $(1 / 20)$ & High $(0 / 20)$ & High $(14 / 20)$ \\
\hline & Medium $(1 / 20)$ & Medium (19/20) & Medium $(1 / 20)$ & Medium $(6 / 20)$ \\
\hline & Slow $(19 / 20)$ & Slow $(0 / 20)$ & Slow $(19 / 20)$ & Slow $(0 / 20)$ \\
\hline \multirow{3}{*}{ Skill } & High $(1 / 20)$ & High $(1 / 20)$ & High $(1 / 20)$ & High $(14 / 20)$ \\
\hline & Medium $(2 / 20)$ & Medium (19/20) & Medium $(16 / 20)$ & Medium $(6 / 20)$ \\
\hline & Low $(17 / 20)$ & Low $(0 / 20)$ & Low $(3 / 20)$ & Low $(0 / 20)$ \\
\hline \multicolumn{5}{|c|}{ Slant/Slope } \\
\hline \multirow{4}{*}{ a)whole writing in general } & Obtuse $(4 / 20)$ & Obtuse $(0 / 20)$ & Obtuse $(1 / 20)$ & Obtuse $(2 / 20)$ \\
\hline & Acute $(4 / 20)$ & Acute $(10 / 20)$ & Acute $(5 / 20)$ & Acute $(4 / 20)$ \\
\hline & Vertical $(10 / 20)$ & Vertical $(5 / 20)$ & Vertical $(12 / 20)$ & Vertical $(7 / 20)$ \\
\hline & Irregular $(2 / 20)$ & Irregular $(5 / 20)$ & Irregular $(2 / 20)$ & Irregular $(6 / 20)$ \\
\hline \multirow{4}{*}{ b)of letters } & Obtuse $(0 / 20)$ & Obtuse $(0 / 20)$ & Obtuse $(1 / 20)$ & Obtuse $(2 / 20)$ \\
\hline & Acute $(2 / 20)$ & Acute $(10 / 20)$ & Acute $(5 / 20)$ & Acute $(3 / 20)$ \\
\hline & Vertical $(1 / 20)$ & Vertical $(5 / 20)$ & Vertical $(11 / 20)$ & Vertical $(6 / 20)$ \\
\hline & Irregular $(17 / 20)$ & Irregular $(5 / 20)$ & Irregular $(4 / 20)$ & Irregular $(9 / 20)$ \\
\hline \multirow{3}{*}{ Size } & Small $(8 / 20)$ & Small $(2 / 20)$ & Small $(8 / 20)$ & Small $(7 / 20)$ \\
\hline & Medium $(7 / 20)$ & Medium $(13 / 20)$ & Medium (9/20) & Medium $(12 / 20)$ \\
\hline & Large $(5 / 20)$ & Large $(5 / 20)$ & Large $(3 / 20)$ & Large $(1 / 20)$ \\
\hline \multicolumn{5}{|c|}{ Space } \\
\hline \multirow{2}{*}{ a)between letters } & Irregular $(20 / 20)$ & Irregular $(3 / 20)$ & Irregular $(6 / 20)$ & Irregular $(15 / 20)$ \\
\hline & Regular $(0 / 20)$ & Regular $(17 / 20)$ & Regular $(14 / 20)$ & Regular (5/20) \\
\hline \multirow{4}{*}{ b)between words } & Normal (2/20) & Normal $(12 / 20)$ & Normal $(9 / 20)$ & Normal $(8 / 20)$ \\
\hline & Narrow $(16 / 20)$ & Narrow $(5 / 20)$ & Narrow $(10 / 20)$ & Narrow (3/20) \\
\hline & Wide $(0 / 20)$ & Wide $(0 / 20)$ & Wide $(1 / 20)$ & Wide $(1 / 20)$ \\
\hline & Variable $(2 / 20)$ & Variable $(3 / 20)$ & Variable $(0 / 20)$ & Variable $(8 / 20)$ \\
\hline
\end{tabular}




\begin{tabular}{|c|c|c|c|c|}
\hline \multirow{4}{*}{ c)between line } & Normal $(8 / 20)$ & Normal $(8 / 20)$ & Normal $(9 / 20)$ & Normal $(1 / 20)$ \\
\hline & Narrow $(6 / 20)$ & Narrow $(2 / 20)$ & Narrow $(8 / 20)$ & Narrow $(2 / 20)$ \\
\hline & Wide $(2 / 20)$ & Wide (2/20) & Wide $(1 / 20)$ & Wide $(8 / 20)$ \\
\hline & Variable $(6 / 20)$ & Variable $(8 / 20)$ & Variable (2/20) & Variable $(9 / 20)$ \\
\hline \multicolumn{5}{|c|}{ Alignment to base line } \\
\hline \multirow{4}{*}{ a)word alignment } & Ascending (1/20) & Ascending (2/20) & Ascending (8/20) & Ascending (15/20) \\
\hline & Descending (3/20) & Descending $(0 / 20)$ & Descending $(1 / 20)$ & Descending $(1 / 20)$ \\
\hline & Horizontal (1/20) & Horizontal $(16 / 20)$ & Horizontal (11/20) & Horizontal (4/20) \\
\hline & Irregular $(15 / 20)$ & Irregular $(2 / 20)$ & Irregular $(0 / 20)$ & Irregular $(0 / 20)$ \\
\hline \multirow{4}{*}{ b)line alignment } & Ascending (4/20) & Ascending $(2 / 20)$ & Ascending (10/20) & Ascending (15/20) \\
\hline & Descending (10/20) & Descending $(0 / 20)$ & Descending $(1 / 20)$ & Descending $(1 / 20)$ \\
\hline & Horizontal (2/20) & Horizontal $(18 / 20)$ & Horizontal $(8 / 20)$ & Horizontal (4/20) \\
\hline & Irregular (4/20) & Irregular $(0 / 20)$ & Irregular $(1 / 20)$ & Irregular $(0 / 20)$ \\
\hline \multirow{3}{*}{ Rhythm } & High $(0 / 20)$ & High $(1 / 20)$ & High $(7 / 20)$ & High $(14 / 20)$ \\
\hline & Medium (3/20) & Medium (19/20) & Medium (11/20) & Medium (6/20) \\
\hline & Low $(17 / 20)$ & Low $(0 / 20)$ & Low $(2 / 20)$ & Low $(0 / 20)$ \\
\hline \multirow{3}{*}{ Line quality } & Good $(0 / 20)$ & Good $(20 / 20)$ & Good $(10 / 20)$ & Good $(3 / 20)$ \\
\hline & Medium (3/20) & Medium $(0 / 20)$ & Medium (6/20) & Medium (15/20) \\
\hline & Poor $(17 / 20)$ & Poor $(0 / 20)$ & Poor $(4 / 20)$ & Poor $(2 / 20)$ \\
\hline \multirow{3}{*}{ Movement } & Finger $(20 / 20)$ & Finger $(0 / 20)$ & Finger $(0 / 20)$ & Finger $(0 / 20)$ \\
\hline & Finger and wrist $(0 / 20)$ & Finger and wrist $(19 / 20)$ & Finger and wrist $(19 / 20)$ & Finger and wrist $(6 / 20)$ \\
\hline & Forearm $(0 / 20)$ & Forearm $(1 / 20)$ & Forearm $(1 / 20)$ & Forearm $(14 / 20)$ \\
\hline \multirow{3}{*}{ Pen pressure } & Heavy $(12 / 20)$ & Heavy $(13 / 20)$ & Heavy $(0 / 20)$ & Heavy $(0 / 20)$ \\
\hline & Medium (7/20) & Medium (6/20) & Medium (6/20) & Medium (5/20) \\
\hline & Light $(1 / 20)$ & Light $(1 / 20)$ & Light $(14 / 20)$ & Light $(15 / 20)$ \\
\hline \multicolumn{5}{|c|}{ Alignment to margins } \\
\hline \multirow{2}{*}{ a)left margin } & Irregular $(16 / 20)$ & Irregular (13/20) & Irregular $(10 / 20)$ & Irregular $(17 / 20)$ \\
\hline & Regular (4/20) & Regular (7/20) & Regular $(10 / 20)$ & Regular $(3 / 20)$ \\
\hline \multirow{2}{*}{ b)right margin } & Irregular $(20 / 20)$ & Irregular $(20 / 20)$ & Irregular $(20 / 20)$ & Irregular $(20 / 20)$ \\
\hline & Regular $(0 / 20)$ & Regular $(0 / 20)$ & Regular $(0 / 20)$ & Regular $(0 / 20)$ \\
\hline \multicolumn{5}{|c|}{ Individual characteristics } \\
\hline \multirow{3}{*}{ Positions of 'i' and 'j' dot } & Just above (18/20) & Just above (10/20) & Just above (16/20) & Just above $(7 / 20)$ \\
\hline & Rightwards (2/20) & Rightwards (8/20) & Rightwards (4/20) & Rightwards (12/20) \\
\hline & Leftwards $(0 / 20)$ & Leftwards $(2 / 20)$ & Leftwards $(0 / 20)$ & Leftwards (1/20) \\
\hline \multirow{4}{*}{ Shape of of 'i' and 'j' dot } & Dotted $(12 / 20)$ & Dotted (5/20) & Dotted $(8 / 20)$ & Dotted (3/20) \\
\hline & Ticked (8/20) & Ticked $(7 / 20)$ & Ticked $(4 / 20)$ & Ticked $(13 / 20)$ \\
\hline & Angular $(0 / 20)$ & Angular $(0 / 20)$ & Angular (5/20) & Angular (4/20) \\
\hline & Circular $(0 / 20)$ & Circular (8/20) & Circular $(3 / 20)$ & Circular $(0 / 20)$ \\
\hline \multirow{3}{*}{$\begin{array}{l}\text { Position of ' } \mathrm{t} \text { ' crossbar } \\
\text { horizontally }\end{array}$} & Leftwards $(20 / 20)$ & Leftwards $(0 / 20)$ & Leftwards $(1 / 20)$ & Leftwards $(0 / 20)$ \\
\hline & Middle (0/20) & Middle $(20 / 20)$ & Middle (19/20) & Middle (14/20) \\
\hline & Rightwards $(0 / 20)$ & Rightwards $(0 / 20)$ & Rightwards $(0 / 20)$ & Rightwards $(6 / 20)$ \\
\hline \multirow{3}{*}{$\begin{array}{c}\text { Position of ' } \mathrm{t} \text { ' crossbar } \\
\text { vertically }\end{array}$} & Upper (4/20) & Upper $(12 / 20)$ & Upper (4/20) & Upper $(11 / 20)$ \\
\hline & Middle (8/20) & Middle (8/20) & Middle (12/20) & Middle $(6 / 20)$ \\
\hline & Lower $(8 / 20)$ & Lower $(0 / 20)$ & Lower $(4 / 20)$ & Lower $(3 / 20)$ \\
\hline
\end{tabular}




\section{Journal of Forensic Sciences \& Criminal Investigation}

\begin{tabular}{|c|c|c|c|c|}
\hline $\begin{array}{l}\text { Capitalisation at abnormal } \\
\text { spaces }\end{array}$ & $\begin{array}{c}\text { Not seen }(0 / 20) \\
\text { Seen }(20 / 20)\end{array}$ & $\begin{array}{c}\text { Not seen }(19 / 20) \\
\text { Seen }(1 / 20)\end{array}$ & $\begin{array}{c}\text { Not seen }(12 / 20) \\
\text { Seen }(8 / 20)\end{array}$ & $\begin{array}{c}\text { Not seen }(6 / 20) \\
\text { Seen }(14 / 20)\end{array}$ \\
\hline Abnormal design of letter & $\begin{array}{c}\text { Not seen }(11 / 20) \\
\text { Seen }(19 / 20)\end{array}$ & $\begin{array}{c}\text { Not seen }(20 / 20) \\
\text { Seen }(0 / 20)\end{array}$ & $\begin{array}{c}\text { Not seen }(11 / 20) \\
\text { Seen }(9 / 20)\end{array}$ & $\begin{array}{c}\text { Not seen }(7 / 20) \\
\text { Seen }(13 / 20)\end{array}$ \\
\hline \multicolumn{5}{|c|}{ Connecting strokes } \\
\hline a)intraword & $\begin{array}{l}\text { Present }(0 / 20) \\
\text { Absent }(12 / 20) \\
\text { Irregular }(8 / 20)\end{array}$ & $\begin{array}{l}\text { Present }(18 / 20) \\
\text { Absent }(1 / 20) \\
\text { Irregular }(1 / 20)\end{array}$ & $\begin{array}{l}\text { Present }(13 / 20) \\
\text { Absent }(5 / 20) \\
\text { Irregular }(8 / 20)\end{array}$ & $\begin{array}{l}\text { Present }(17 / 20) \\
\text { Absent }(0 / 20) \\
\text { Irregular }(3 / 20)\end{array}$ \\
\hline b)interword & $\begin{array}{l}\text { Present }(0 / 20) \\
\text { Absent }(20 / 20)\end{array}$ & $\begin{array}{l}\text { Present }(0 / 20) \\
\text { Absent }(20 / 20)\end{array}$ & $\begin{array}{l}\text { Present }(0 / 20) \\
\text { Absent }(20 / 20)\end{array}$ & $\begin{array}{l}\text { Present }(1 / 20) \\
\text { Absent }(19 / 20)\end{array}$ \\
\hline Correction & $\begin{array}{c}\text { Not seen }(4 / 20) \\
\text { Seen }(16 / 20)\end{array}$ & $\begin{array}{c}\text { Not seen }(19 / 20) \\
\text { Seen }(1 / 20)\end{array}$ & $\begin{array}{c}\text { Not seen }(17 / 20) \\
\text { Seen }(3 / 20)\end{array}$ & $\begin{array}{c}\text { Not seen }(8 / 20) \\
\text { Seen }(12 / 20)\end{array}$ \\
\hline Overwriting & $\begin{array}{l}\text { Not seen }(4 / 20) \\
\text { Seen }(16 / 20)\end{array}$ & $\begin{array}{c}\text { Not seen }(12 / 20) \\
\text { Seen }(8 / 20)\end{array}$ & $\begin{array}{c}\text { Not seen }(16 / 20) \\
\text { Seen }(4 / 20)\end{array}$ & $\begin{array}{c}\text { Not seen }(11 / 20) \\
\text { Seen }(9 / 20)\end{array}$ \\
\hline Obliteration & $\begin{array}{c}\text { Not seen }(9 / 20) \\
\text { Seen }(11 / 20)\end{array}$ & $\begin{array}{c}\text { Not seen }(15 / 20) \\
\text { Seen }(5 / 20)\end{array}$ & $\begin{array}{c}\text { Not seen }(12 / 20) \\
\text { Seen }(8 / 20)\end{array}$ & $\begin{array}{c}\text { Not seen } 1(0 / 20) \\
\text { Seen }(10 / 20)\end{array}$ \\
\hline \multicolumn{5}{|c|}{ Omission } \\
\hline $\begin{array}{c}\text { a)omission of strokes from } \\
\text { letter }\end{array}$ & $\begin{array}{c}\text { Not seen }(14 / 20) \\
\text { Seen }(6 / 20)\end{array}$ & $\begin{array}{c}\text { Not seen }(20 / 20) \\
\text { Seen }(0 / 20) \\
\end{array}$ & $\begin{array}{c}\text { Not seen }(18 / 20) \\
\text { Seen }(2 / 20)\end{array}$ & $\begin{array}{c}\text { Not seen }(6 / 20) \\
\text { Seen }(14 / 20)\end{array}$ \\
\hline $\begin{array}{l}\text { b)omission of letter from } \\
\text { word }\end{array}$ & $\begin{array}{c}\text { Not seen }(9 / 20) \\
\text { Seen }(11 / 20)\end{array}$ & $\begin{array}{c}\text { Not seen }(20 / 20) \\
\text { Seen }(0 / 20)\end{array}$ & $\begin{array}{c}\text { Not seen }(17 / 20) \\
\text { Seen }(3 / 20)\end{array}$ & $\begin{array}{c}\text { Not seen }(6 / 20) \\
\text { Seen }(14 / 20)\end{array}$ \\
\hline c)omission of word & $\begin{array}{c}\text { Not seen }(14 / 20) \\
\text { Seen }(6 / 20)\end{array}$ & $\begin{array}{c}\text { Not seen }(20 / 20) \\
\text { Seen }(0 / 20)\end{array}$ & $\begin{array}{c}\text { Not seen }(19 / 20) \\
\text { Seen }(1 / 20)\end{array}$ & $\begin{array}{c}\text { Not seen }(20 / 20) \\
\text { Seen }(0 / 20)\end{array}$ \\
\hline d)omission of line & $\begin{array}{c}\text { Not seen }(14 / 20) \\
\text { Seen }(6 / 20)\end{array}$ & $\begin{array}{c}\text { Not seen }(20 / 20) \\
\text { Seen }(0 / 20)\end{array}$ & $\begin{array}{c}\text { Not seen }(18 / 20) \\
\text { Seen }(2 / 20)\end{array}$ & $\begin{array}{c}\text { Not seen }(20 / 20) \\
\text { Seen }(0 / 20)\end{array}$ \\
\hline Misspellings & $\begin{array}{c}\text { Not seen }(20 / 20) \\
\text { Seen }(0 / 20)\end{array}$ & $\begin{array}{c}\text { Not seen }(10 / 20) \\
\text { Seen }(10 / 20)\end{array}$ & $\begin{array}{c}\text { Not seen }(12 / 20) \\
\text { Seen }(8 / 20)\end{array}$ & $\begin{array}{c}\text { Not seen }(14 / 20) \\
\text { Seen }(6 / 20)\end{array}$ \\
\hline Punctuation marks & $\begin{array}{c}\text { Present }(12 / 20) \\
\text { Absent }(8 / 20)\end{array}$ & $\begin{array}{c}\text { Present }(20 / 20) \\
\text { Absent }(0 / 20)\end{array}$ & $\begin{array}{c}\text { Present }(19 / 20) \\
\text { Absent }(1 / 20)\end{array}$ & $\begin{array}{l}\text { Present }(20 / 20) \\
\text { Absent }(0 / 20)\end{array}$ \\
\hline Lateral expansion & $\begin{array}{l}\text { Present }(0 / 20) \\
\text { Absent }(20 / 20)\end{array}$ & $\begin{array}{l}\text { Present }(1 / 20) \\
\text { Absent }(19 / 20)\end{array}$ & $\begin{array}{l}\text { Present }(0 / 20) \\
\text { Absent }(20 / 20)\end{array}$ & $\begin{array}{l}\text { Present }(10 / 20) \\
\text { Absent }(10 / 20)\end{array}$ \\
\hline Abbreviations & $\begin{array}{c}\text { Not seen }(20 / 20) \\
\text { Seen }(0 / 20)\end{array}$ & $\begin{array}{l}\text { Not seen }(20 / 20) \\
\text { Seen }(0 / 20)\end{array}$ & $\begin{array}{c}\text { Not seen }(20 / 20) \\
\text { Seen }(0 / 20)\end{array}$ & $\begin{array}{c}\text { Not seen }(3 / 20) \\
\text { Seen }(17 / 20)\end{array}$ \\
\hline
\end{tabular}

Depending on the sample size (80), each characteristic for each occupation is observed individually and then the inter-comparison of such characteristics is done to see the similarities and dissimilarities between the writing of people indulged in different occupations.

Table 2: Chi-Value of each class and individual characteristics

\begin{tabular}{|c|c|c|}
\hline S.No. & Characteristics & Chi-Value \\
\hline & General characteristics & 41.4444 \\
\hline 1. & Type of lettering & 100.0744 \\
\hline 2. & Legibility & 85.9766 \\
\hline 3. & Speed & 14.1277 \\
\hline 4. & Slant & 9.3224 \\
\hline 5. & Size & 33.6547 \\
\hline 6. & Spacing & \\
\hline a) & Between letters & \\
\hline
\end{tabular}


Journal of Forensic Sciences \& Criminal Investigation

\begin{tabular}{|c|c|c|}
\hline b) & Between words & 24.6974 \\
\hline c) & Between lines & 24.4649 \\
\hline 7. & Alignment to baseline & \\
\hline (a) & Word alignment & 65.137 \\
\hline (b) & Line alignment & 50.0161 \\
\hline 8. & Rhythm & 41.0627 \\
\hline 9. & Line quality & 65.7839 \\
\hline 10. & Movement & 89.0066 \\
\hline 11. & Pen pressure & 39.5035 \\
\hline 12. & Alignment to margin & 7.1429 \\
\hline \multicolumn{3}{|c|}{ Individual characteristics } \\
\hline 13. & Position of ' $i$ ' and ' $j$ ' dots & 14.4994 \\
\hline 14. & Shape of ' $i$ ' and ' $j$ ' dots & 21.3237 \\
\hline 15. & Position of ' $\mathrm{t}$ ' crossbar horizontally & 62.3164 \\
\hline 16. & Position of ' $t$ ' crossbar vertically & 34.139 \\
\hline 17. & Capitalisation at abnormal spaces & 36.2907 \\
\hline 18. & Abnormal design of letter & 15.3162 \\
\hline 19. & Correction & 32.0833 \\
\hline 20. & Overwriting & 4.8391 \\
\hline 21. & Obliteration & 4.2967 \\
\hline 22. & Omission of strokes & 25.5683 \\
\hline 23. & Omission of letters & 25.2603 \\
\hline 24. & Misspellings & 24.1561 \\
\hline
\end{tabular}

Chi-square test was done using online calculator to find the chi-value helpful in identifying the ability of a characteristic to differentiate the writers with different occupations.

(Chi-square test was done using online calculator to find the chi-value helpful in identifying the ability of a characteristic to differentiate the writers with different occupations)In general characteristics, legibility, speed, alignment to baseline(word and line alignment), rhythm, line quality, movement were found significant characteristics to differentiate between different occupations, while type of lettering, spacing (between word, letters and line), pen pressure were found moderately significant while alignment to margins, size, slant were insignificant. In individual characteristics, shape of I and j dot, position t-crossbar horizontally were found highly significant while position of t-crossbar vertically, correction, misspellings, omission of strokes and letter, capitalization at abnormal spaces, and abnormal design of letter were found moderately significant and some characteristics such as overwriting, obliteration were insignificant.

\section{Conclusion}

The present study indicates that the handwriting of the writer and educational background are interrelated. Doctors' handwriting was found to be highly skilful and rhythmic showing high educational background which shows their higher graphic maturity as compared to unskilled workers, teachers and bankers. While handwriting samples of teachers and bankers were found to be moderately skilful which shows their moderate educational level. The handwriting of unskilled workers was found poor showing their low educational background mostly below 10th grade which in turn shows their low graphic maturity and lack of writing habit on daily basis. The degree of similarity $\%$ shows that certain characteristics were prominently adapted based on occupation such as cursive handwriting was found to be $90 \%$ in the handwriting samples of teachers (1st to 5 thgrade) because they teach usually cursive handwriting in schools. Similarly legibility of doctor's handwriting was found poor $(90 \%)$ as their speed and skill of handwriting is high. The type of lettering in unskilled workers was found to be 'printed' (90\%) due to their low skill and low speed and low education level. It may be said that this study validated the alternate hypothesis with $75 \%$ percent confidence.

\section{Limitations}

a) The graphic maturity of the writer don't solely depends upon the education level that is if a person is not that highly educated but his work involves a lot of writing then he will achieve his graphic maturity much earlier.

b) In case of few occupations such as Bankers, they have different educational background so this a limiting factor in evaluating the samples.

c) The time since working in a particular occupation is a major factor that is the working experience the more 
adaptations are seen in the handwriting samples of participants and vice-versa.

d) The frequent changes in the different occupations are also major limitation but in-depth analysis will help to characterise the same.

\section{References}

1. Corney MW (2003) Analysing E-mail text authorship for forensic purpose. Handwriting in early childhood.

2. Srihari S N, Cha SH, Arora H (2011) Individuality of Handwriting.

3. Hilton O (1982) Scientific Examination of Questioned Documents. New York: Elsevier Science Publishing Co

4. Michell, Colin Simon (2013) Investigating the use of forensic stylistic and stylometric techniques in the analyses of authorship on a publicly accessible social networking site.

5. Ellen D, Stephen P Day, Christopher Dvais (2005) Scientific Examination of Documents: Methods and Techniques, Third Edition. Boca Raton: CRC Press, USA.

6. Berent S (1974) Field dependence and performance on a writing task: Perceptual and Motor Skills 38(2): 65.

7. Bhavana Desai, J L Kalyan (2013) Forensic Examination of Handwriting And Signatures. International journal of innovative research and development 2(5).
8. Khushbukumari (2015) Handwriting characteristics variability among populations of Daman and Diu. Journal of international academic research for multidisciplinary 3(4).

9. William L Leaver (2006) Introduction to Forensic Document Examination pp. 223-248.

10. Drempt V, McCluskey, Lannin (2011) A review of factors that influence adult handwriting performance: Australian occupational therapy journal 58(5): 321-398.

11. Franzsen D, Stewart A (2014) Identifying the factors that contribute to handwriting problems experienced by students at a higher education institution in South Africa: South Africa journal of occupational therapy $44(1): 3-8$.

12. Baker KD, Tan TN, HES (2000) Personal identification based on handwriting: The journal of the pattern recognition society 33(1): 149-160.

13. Akoria OA, Isha AO (2008) Prescription writing in public and private hospitals in Benin city, Nigeria: The effects of an educational intervention: The Canadian journal of clinical pharmacology 15(2): 295-305.

14. Graham S, Weintraub N, Berninger V W (1998) The relationship between handwriting style and speed and legibility: Journal of Educational Research 91(5): 290-296.

\section{Your next submission with Juniper Publishers will reach you the below assets}

- Quality Editorial service

- Swift Peer Review

- Reprints availability

- E-prints Service

- Manuscript Podcast for convenient understanding

- Global attainment for your research

- Manuscript accessibility in different formats ( Pdf, E-pub, Full Text, Audio)

- Unceasing customer service

Track the below URL for one-step submission https://juniperpublishers.com/online-submission.php 